\title{
Disruptive photonic technologies for the future sustainable high-capacity metro network
}

\author{
Alberto Gatto ${ }^{1}$, Paola Parolari ${ }^{1}$, Mariangela Rapisarda ${ }^{1}$, Christian Neumeyr $^{2}$, Srivathsa Bhat ${ }^{3}$, \\ Giovanni Delrosso ${ }^{3}$, Michela Svaluto Moreolo ${ }^{4}$, Josep M. Fabrega ${ }^{4}$, Laia Nadal $^{4}$, Pierpaolo Boffi ${ }^{1 *}$ \\ ${ }^{I}$ POLITECNICO DI MILANO, Dept. Electronics, Information and Bioengineering, \\ PoliCom Lab, Via G. Ponzio 34/5, 20133 Milan (ITALY) \\ ${ }^{2}$ Vertilas GmbH, Garching, Germany \\ ${ }^{3}$ VTT Technical Research Centre of Finland Ltd, Espoo, Helsinki, Finland. \\ ${ }^{4}$ Centre Tecnologic de Telecomunicacions de Catalunya (CTTC/CERCA), Castelldefels, Barcelona, Spain \\ *pierpaolo.boffi@polimi.it
}

\begin{abstract}
An original technological solution based on the exploitation of long-wavelength VCSELs directly-modulated with multicarrier modulation formats is proposed to implement multi- $\mathrm{Tb} / \mathrm{s}$ transceivers for the next-generation metropolitan area network. The modular approach conceived for the design and realization of the transmitter guarantees scalability and low cost, low power consumption and reduced footprint.
\end{abstract}

Keywords: optical communications, VCSEL, DMT, S-BVT, metro networks.

\section{INTRODUCTION}

Nowadays, most of the total data traffic is concentrated in less of the $5 \%$ of the geographical area, for example in the metropolitan area networks (MANs). With respect to the long-haul transport scenario, for this kind of networks the request of low cost, low power consumption and reduced footprint is mandatory, in addition to the demand of a huge throughput to be transported. Innovative tailored photonic technologies have to be developed, completely new compared to the traditional high-capacity approach adopted for the long-haul fibre infrastructure, considered too expensive and power hungry to realize a future MAN. Different solutions are proposed in literature to guarantee very high transmission capacity, for example based on the exploitation of ultra-high speed data rate per carrier [1] achieved thanks to silicon-photonics ( $\mathrm{SiPh}$ ) devices or of ultra-wide band amplification ( $\mathrm{S}, \mathrm{C}$ and $\mathrm{L}$ band) to support hundreds of wavelength-division multiplexed (WDM) channels [2].

To match the challenges of the future sustainable MAN, the Horizon 2020 project PASSION [3] proposes an alternative approach by developing a new transmitter (TX), exploiting the capabilities of long-wavelength vertical cavity surface emitting lasers (VCSELs) emitting in the $\mathrm{C}$ band, directly modulated (DM) by multicarrier formats, such as discrete multitone (DMT). Dense wavelength-division multiplexed (WDM) VCSELs are integrated in a silicon-on-insulator (SOI) module (MOD) to provide up to $2-\mathrm{Tb} / \mathrm{s}$ capacity. A modular approach is used in the design of a super-MOD, in order to build a sliceable bandwidth/bitrate variable transceiver (S-BVT) $[4,5]$, providing $25-\mathrm{GHz}$ fine granularity and guaranteeing multi- $\mathrm{Tb} / \mathrm{s}$ capacities, according to the requested MAN traffic scaling.

\section{Long-wavelength high-bandwidth VCSEL}

Nowadays, short-wavelength multi-mode VCSELs in GaAs are massively employed for high-speed data communications. The benefits of VCSELs in terms of cost, testability and consumption [6] are well known. To face the continuous demand of bandwidth and connection reach, such as in case of MAN, it is necessary to exploit VCSELs [7] characterized by: (i) Single-mode operation to limit the effect of modal and chromatic dispersion; (ii) Long-wavelength emission in the $\mathrm{C}$ band to exploit dense WDM; (iii) High bandwidth to achieve very high transmission rates. For the realization of PASSION S-BVT, we employ VCSEL sources produced in InP by VERTILAS GmbH: single-mode operation is achieved by an appropriate transverse waveguide structure with sidemode suppression ratio higher than $35 \mathrm{~dB}$ and a stable polarization output. Long-wavelength emission in the $\mathrm{C}$ band is assured by exploiting buried tunnel junction (BTJ) approach. The reduced active area allows an expected far field confined in less than $12^{\circ}$ with a structure optimized for massive integration. A short-cavity is obtained by a very short resonator length and optimized active region, guaranteeing high bandwidth (above $18 \mathrm{GHz}$ ). This performance is combined to very low power consumption, less than $30 \mathrm{~mW}$. Fig. 1 shows the short-cavity structure optimized for high modulation bandwidth (on the left). On the right, a picture of the VERTILAS long-wavelength VCSEL employed in the realization of the S-BVT device. 

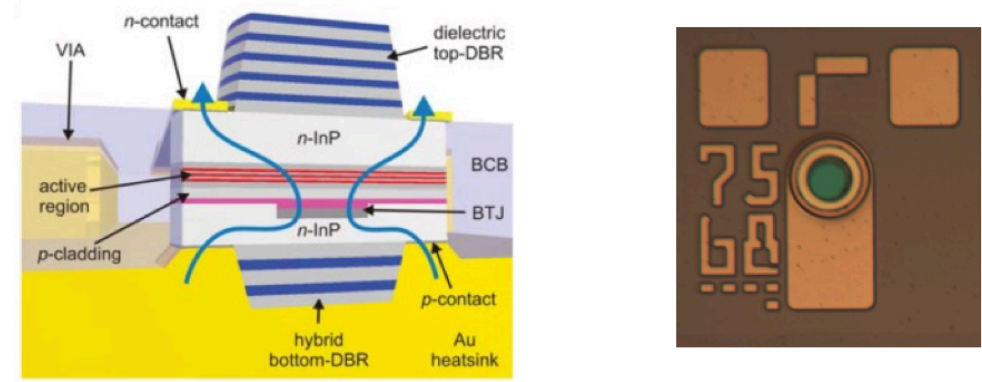

Figure 1. Short-cavity VCSEL structure optimized for high modulation bandwidth (on the left) and picture of the VERTILAS long-wavelength VCSEL.

\section{DMT modulation for high capacity per VCSEL}

DMT is a very effective multicarrier modulation technique to increase the transported capacity, matching the nonuniform and bandwidth-limited response of the whole communication system [8]. Very high bit rate transmission per single wavelength can be achieved thanks to the exploitation of bit and power loading, also in case of direct modulation of the optical laser source [9]. DMT is adopted for the modulation of the long-wavelength InP VCSELs described in Section 2 to perform at least $50 \mathrm{~Gb} / \mathrm{s}$ rate per directly-modulated VCSEL. Fig. 2 presents preliminary results obtained with a C-band VCSEL characterized by 14-GHz modulation bandwidth: more than $50 \mathrm{~Gb} / \mathrm{s}$ rate is experimentally demonstrated thanks to DMT modulation by exploiting just $10-\mathrm{GHz}$ electrical bandwidth of the modulation signal in case of dual side band modulation. The DMT signal is composed by 256 sub-carriers in 10 $\mathrm{GHz}$ range, i.e. the sub-carrier spacing is $39.0625 \mathrm{MHz}$. A cyclic prefix of about $2.1 \%$ of the symbol length is added. The bias current is set at $9 \mathrm{~mA}$, with an optimized $10-\mathrm{mA}$ modulation amplitude to limit the frequency chirp effect. 5-fold improvement of the transported capacity is obtained with respect to the electrical modulation bandwidth, thanks to directly-modulated DMT. In order to increase the spectral efficiency, while providing a higher resilience towards filtering effects, single side band modulation can be also employed [10].
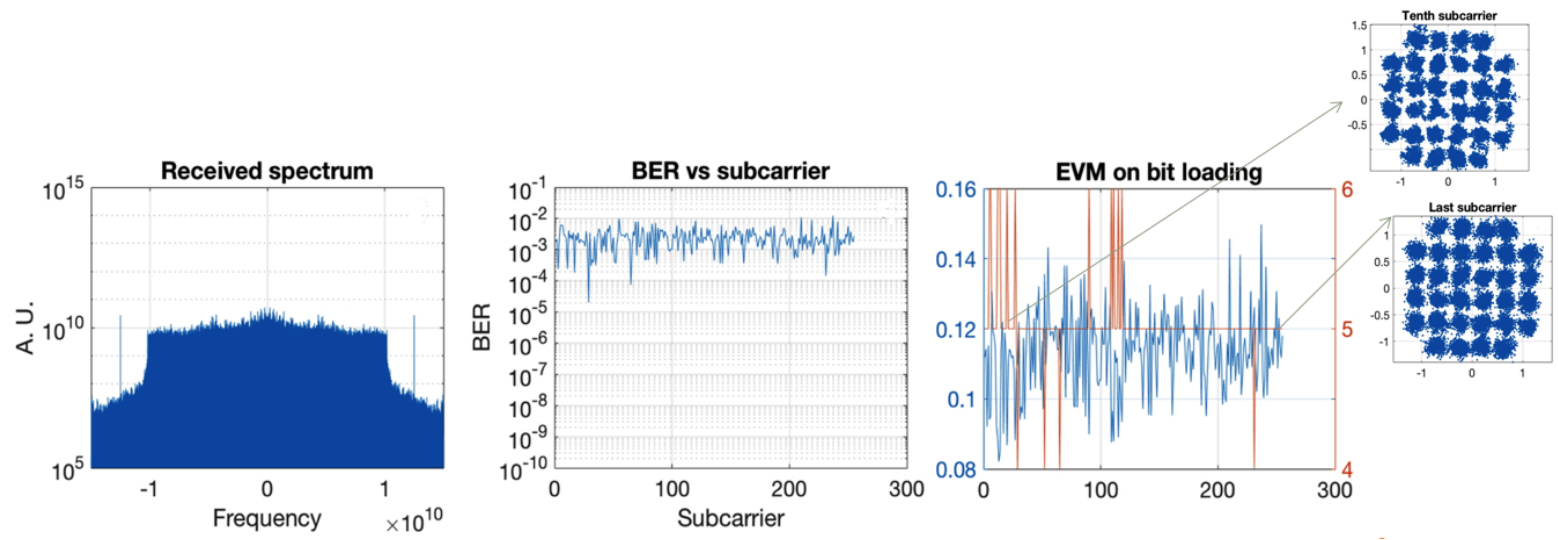

Figure 2. Results for DMT directly-modulated long-wavelength VCSEL: a) received electrical spectrum; b) BER per subcarrier; c) bit loading and corresponding EVM. In the in-sets examples of received constellations for the tenth and the last subcarrier. Capacity of $50.2 \mathrm{~Gb} / \mathrm{s}$ is achieved targeting BER less than $3.8 \cdot 10^{-3}$.

\section{PASSION modular S-BVT scheme}

PASSION project is developing an innovative photonic TX based on the VERTILAS VCSELs described in Section 2. The employed sources emit in the C band, covering the ITU channels from 19 to 59, with dense WDM spacing of $25 \mathrm{GHz} .50 \mathrm{~Gb} / \mathrm{s}$ rate is achieved per state of polarization (SOP) per VCSEL thanks to DMT, as described in Sec.3. A 40-VCSEL based MOD is designed by integrating in SIPh 4 sub-MODs, each one containing 10 VCSELs with 400-GHz spacing, as shown in Fig. 3. In the MOD, the 40 50-Gb/s modulated VCSEL emission wavelengths cover the $\mathrm{C}$ band with $100-\mathrm{GHz}$ granularity, so an aggregated capacity per MOD of up to $2 \mathrm{~Tb} / \mathrm{s}$ is achieved. This 40-VCSELs MOD constitutes the PASSION fundamental building block for the TX development. By combining 4 of such a MOD, a full 160 -channels TX super-MOD is obtained, characterized by $25-\mathrm{GHz}$ granularity and enabling up to $8 \mathrm{~Tb} / \mathrm{s}$ total transmitted capacity (Fig. 3).

The modular approach allows to design and develop identical MODs to build the super-MOD: the 40 emitted wavelengths from the VCSELs in each MOD are fine-tuned in a range of 0-75 GHz through the VCSEL bias current and stabilized by a temperature cooler (as shown in Figure 4). The exploited modularity offers the ability to fabricate and stock only one MOD type and to use the identical 40-channel MODs to build the full 160-channel TX super-MOD. 


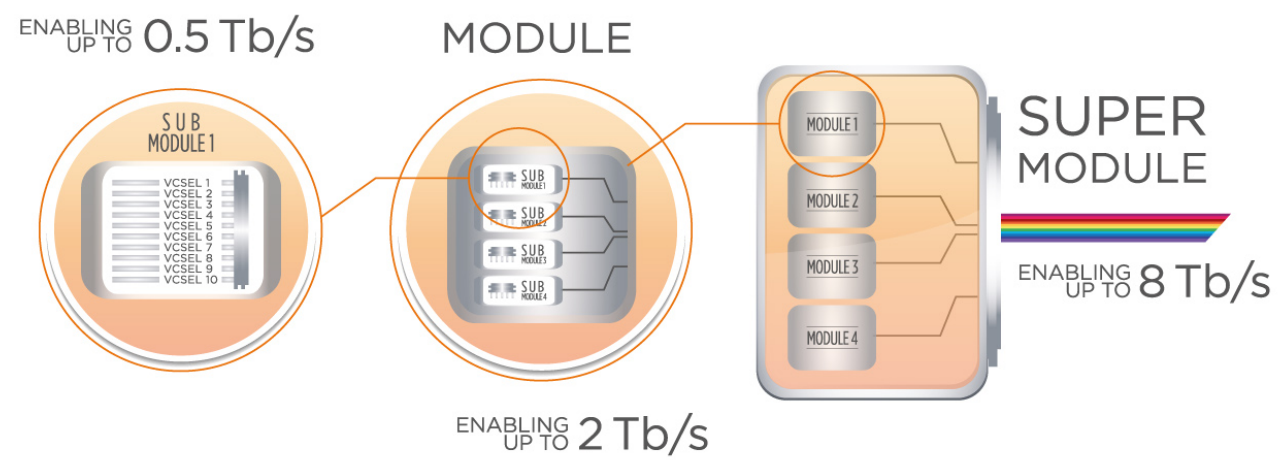

Figure 3. PASSION S-BVT TX structure based on a modular approach.

In applications regarding MANs, polarization-division multiplexing (PDM) can be also exploited combined to coherent detection at the receiver side, coupling the outputs of 2 identical super-MODs, orthogonal in SOP, achieving up to $16 \mathrm{~Tb} / \mathrm{s}$ capacity. Coherent detection allows not only to demultiplex the SOPs, but also to compensate the chromatic dispersion in case of significant reaches, also hundreds of km long, typical of MANs. In this way, the designed modular S-BVT [11] can adaptively load/manipulate the spectrum with 25-GHz fine granularity, exploiting multicarrier modulation, and multiple dimensions, including the C-band spectrum and the polarization [12].

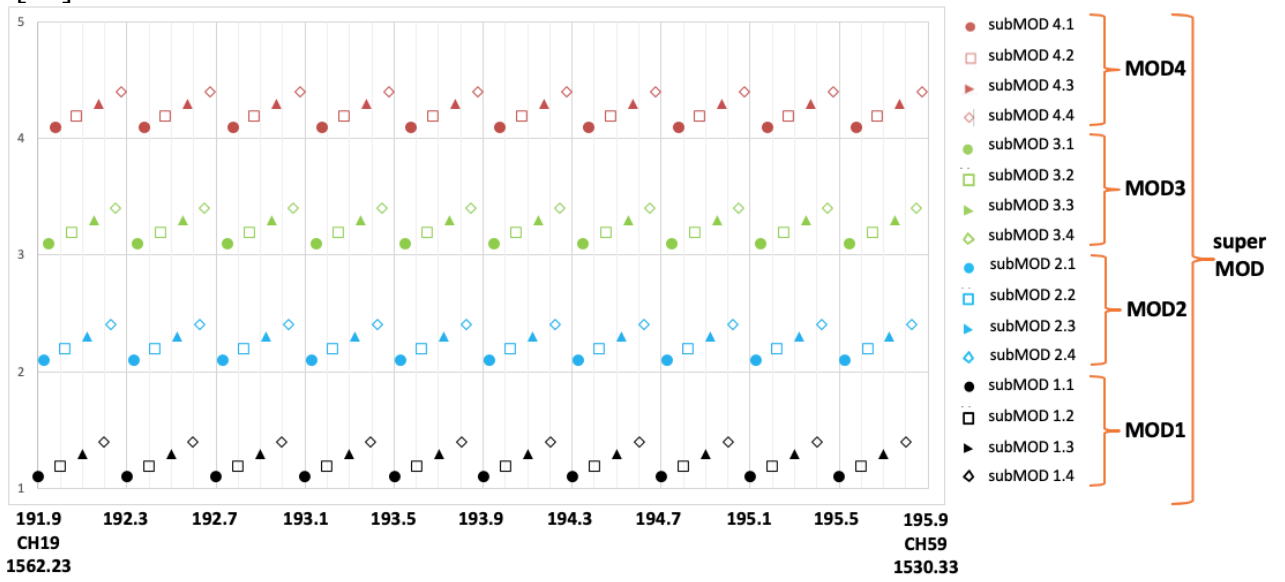

Figure 4. Grid of the VCSEL emission wavelengths for the 4 TX MODs constituting the super-MOD designed according to a modular approach.

\section{PASSION transmitter development}

The scheme of the TX MOD combining $40100 \mathrm{GHz}$ spaced VCSELs is shown in Fig. 5 on the left. The SOI photonic integrated chip (PIC) of the MOD is fabricated as part of the VTT MPW6 process run [13]: its design includes 4 arrayed waveguide gratings (AWGs) to multiplex 10 VCSEL channels into a single output waveguide in each sub-MOD (with very low loss and crosstalk: $1.2 \mathrm{~dB}$ and $-35 \mathrm{~dB}$, respectively) and a 2-stage MZI interleaver to combine the 4 sub-MOD 10-channels waveguide outputs into a single 40-channels multiplexed output waveguide. Integrated optical $45^{\circ}$ TIR mirrors on Si-PIC in up-reflecting configuration are employed for VCSEL$\mathrm{Si}$ waveguide coupling, as shown in Fig. 5 on the right. High efficiency is achieved with coupling loss lower than $0.5 \mathrm{~dB}$. To electrically interconnect the SOI PIC with the 40 VCSEL sources, an electrical interposer based on a land grid array (LGA) pad architecture is adopted. Taking into account the channel pitch depending on the dimension of the VCSELs and the linear drivers, the achieved TX chip size is about $4 \mathrm{~cm}^{2}$ for an emitted capacity of $2 \mathrm{~Tb} / \mathrm{s}$. Moreover, power consumption is maintained less than $5 \mathrm{pJ} / \mathrm{bit}$. Fig. 6 shows the PIC design (on the left) and the realized SOI PIC (on the right).

\section{Conclusion}

We have presented the photonic solutions developed in PASSION project to provide a disruptive VCSEL-based TX sustainable in terms of cost, power consumption and footprint. The exploitation of high-bandwidth longwavelength directly DMT modulated VCSELs allows to reach up to $2 \mathrm{~Tb} / \mathrm{s}$ emitted capacity per module. Thanks to the adopted modular approach, the same module can be employed in the realization of a multi-Tb/s S-BVT operating with $25-\mathrm{GHz}$ granularity facing the request of a huge throughput and traffic scaling of the future MAN. 

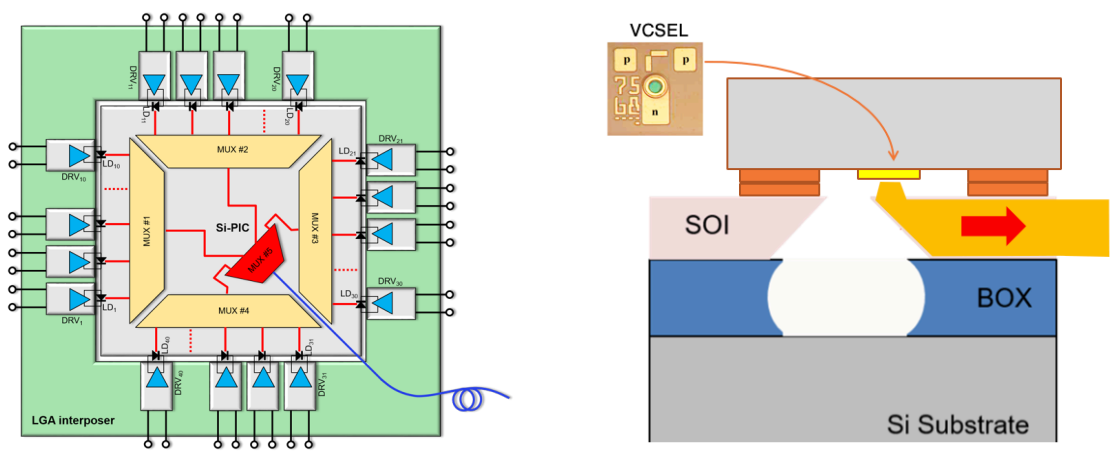

Figure 5. Scheme of the 40-VCSELs module providing a total capacity of $2 \mathrm{~Tb} / \mathrm{s}$ (on the left). VCSEL-waveguide coupling by $45^{\circ}$ TIR mirror in up-reflecting configuration (on the right).
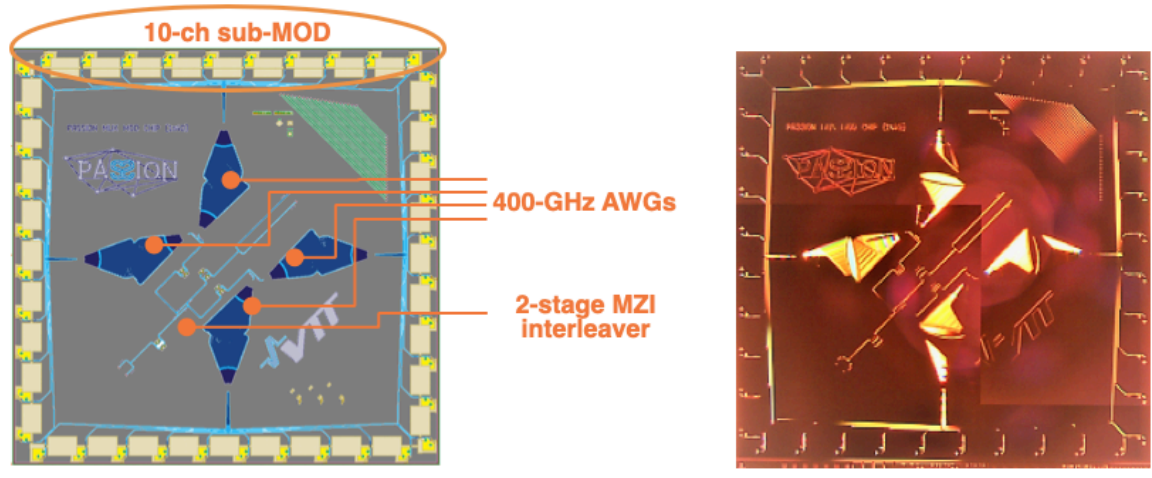

Figure 6. 40-VCSELs module design (on the left) and realized SOI PIC (on the right).

\section{ACKNOWLEDGEMENTS}

This work has been supported by the H2020 EU PASSION Project (GA 780326).

\section{REFERENCES}

[1] J. Lin, et al. "Joint Digital and Optical Pre-compensation for 720 Gb/s All-Silicon IQ modulator Single Carrier Transmission", Proc. ECOC 2018, paper We3G.2 (2018).

[2] J. Renaudier, "100nm ultra-wide optical fiber transmission systems using semiconductor optical amplifiers", Proc. ECOC 2018, paper Mo4G.5 (2018).

[3] www.passion-project.eu

[4] M. Svaluto Moreolo, et al. "SDN-enabled sliceable BVT based on multicarrier technology for multi-flow rate/distance and grid adaptation." J. Lightwave Technol., 34, 1516-1522 (2016).

[5] M. Svaluto Moreolo, et al., "VCSEL-based sliceable bandwidth/bitrate variable transceivers," in SPIE-PWO, San Francisco, CA (USA), 2019.

[6] A. Boletti, et al., "Performance analysis of communication links based on VCSEL and silicon photonics technology for high-capacity data-intensive scenario", Optics Express, 23, 1806-1815 (2015)

[7] A. Gatto, et al., "Very high-capacity short-reach VCSEL systems exploiting multicarrier internsity modulation and direct detection", Optics Express, 24, 12, 12769-12775 (2016)

[8] A. Gatto, et al., "FDM and DMT performance comparison in high capacity point-to-poiny fibre links for intra/inter-datacentre connections," Optical Fiber Technology, 42, 92-96 (2018).

[9] A. Gatto, et al., "Beyond $25 \mathrm{~Gb} / \mathrm{s}$ Directly-Modulated Widely Tunable VCSEL for Next Generation Access Network,"in Proceedings OFC 2018, paper Th1E.2 (2018).

[10] M. Rapisarda, et al. "Impact of Chirp in High-Capacity Optical Metro Networks Employing DirectlyModulated VCSELs," Photonics, 5, 51 (2018).

[11] M. Svaluto Moreolo et al., "Modular SDN-enabled S-BVT Adopting Widely Tunable MEMS VCSEL for Flexible/Elastic Optical Metro Networks," in Proceedings OFC 2018, paper M1A.7 (2018).

[12] M. Svaluto Moreolo et al., "Programmable VCSEL-based transceivers for multi-terabit capacity networking," in Proc. CLEO 2019, San José, CA, USA (2019).

[13] S. Bhat, M. Harjanne, F. Sun, et al. "Low Loss Devices fabricated on the Open Access 3mm SOI Waveguide Platform at VTT", Proc. ECIO 2019, paper T.Po2.18 (2019). 